\title{
Human Plague Case Diagnosed in Ningxia Tracked to Animal Reservoirs - Inner Mongolia Autonomous Region, China, 2021
}

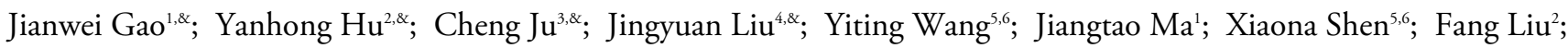 \\ Jin Guo ${ }^{5,6}$; Xinxin $\mathrm{Yu}^{2}$; Wen Zhang, ${ }^{5,6}$ Shuyi Wang'; Kun Lii, ${ }^{5,6}$ Zhongbing Zhang'; Biao Kan ${ }^{5,6}$; Wenrui Wang \\ Xianbin Cong'; Mengguang Fan ${ }^{2}$; Wei Li ${ }^{5,6}$; Kuidong Shao ${ }^{3, *}$; Tao Zhang ${ }^{1, * *}$; Jianyun Li ${ }^{2, *}$; Yumeng Wang ${ }^{5,6, *}$
}

\section{Summary \\ What is already known about this topic? \\ There were a total of 4 and 3 human plague cases that occurred in the Inner Mongolia Autonomous Region in 2019 and 2020, respectively, with 1 and 2 deaths in 2019 and 2020 respectively, which indicated that plague still poses a significant threat to human health especially for farmers, shepherds, or residents living in native plague foci. \\ What is added by this report? \\ On August 14, 2021, 1 patient from the Otog Qi (County) in the Inner Mongolia sought treatment in Yinchuan City (the capital of Ningxia Hui Autonomous Region), where the patient was diagnosed with bubonic plague and secondary septicemic plague. The genetic source tracking of associated Yersinia pestis strains indicated that human plague cases were infected from animal reservoirs in Inner Mongolia. \\ What are the implications for public health practice? \\ Major threats of plague to residents living in native plague foci are the infection by bites of bacterium- bearing fleas or direct contact with diseased or dead plague-infected animals. And the ability of early diagnostic is very critical for county-level hospital in native plague foci.}

On August 20, 2021, a human plague case (55-yearold woman) suffered bubonic plague with secondary septicemic plague was reported from General Hospital of Ningxia Medical University (GHNMU) in Ningxia Hui Autonomous Region (Ningxia), China. Effective patient's treatment, together with enhanced rodents plague surveillance and control, was performed in Ningxia and Inner Mongolia Autonomous Region (Inner Mongolia). The genome-wide single nucleotide polymorphism (SNP) analysis was used in source tracing based on phylogenetic relationship of Yersinia pestis ( $Y$. pestis) strains in this event. The $Y$. pestis strain isolated from the patient in Ningxia and the strain from local Meriones unguiculatus (M. unguiculatus) that were found near the patients' residences were clustered into the same lineage (2.MED3q). Such observations indicated that human plague cases originated from local reservoirs.

\section{INVESTIGATION AND RESULTS}

On August 20, 2021, a human plague case suffering from bubonic plague with secondary septicemic plague was reported from GHNMU in Ningxia. The patient was a 55-year-old female herdsman who lived in Wulan Village, Otog Qi (County), Erdos City in Inner Mongolia. On August 14 and 15, 2021, the patient presented with the onset of nausea and vomiting with low blood pressure and sought treatment in a village clinic but the patient's condition deteriorated further. On August 16, 2021, the patient was admitted to the local county hospital in Pingluo County of Ningxia for high fever $\left(40{ }^{\circ} \mathrm{C}\right)$ with weakness and vomiting. Subsequently, on August 17 later, the patient was transferred to GHNMU in Yinchuan City and was admitted to the intensive care unit (ICU) for septic shock symptoms with left inguinal lymphadenitis. During this period, no coughing, chest pain, or breathlessness was observed. Thereafter, the blood of patient was conducted a bacteria culture and examined through biochemical analyzer in GHNMU, and the results of biochemical analyzer reported that bacteria in the patient's blood was Yersina genus. Such results led the clinical doctors in GHNMU began to suspect the patient might suffer from plague and reported to the Ningxia CDC on August 20.

This human plague case was confirmed via polymerase chain reaction (PCR) positive results in lymph node aspirates and blood by the Ningxia CDC within four hours, targeting the cafl, pla, and 
YPO0392 genes of $Y$. pestis (1), as well as the positive results of the colloidal gold-immunochromatography assay and reverse indirect hemagglutination assay (RIHA) test targeting the F1 antigen in lymph node aspirates and blood. The titer of RIHA for the lymph node aspirates and blood were 1:64 and 1:128, respectively. In addition, the bacteria isolated from blood were identified as $Y$. pestis by Gram staining, microscopy, and a phage lysis test. While, the PCR assays for the patient were negative in the sputum and throat specimens of the patient.

Based on clinical manifestations and laboratory test results, the patient was diagnosed with bubonic plague with secondary septicemic plague and she was treated with antibiotics (streptomycin and ciprofloxacin) and recovered on September 6, 2021.

One $Y$. pestis (Ningxia 2021) isolated from the 55year-old patient was sequenced by Ningxia CDC. Meanwhile, one strain (Neimeng 2021) isolated from $M$. unguiculatus eighty meters away from the house of the patient was isolated and sequenced by Inner Mongolia CDC. Under the source tracing mechanism of the Chinese Pathogen Identification Net (CPIN), the patient-related (Ningxia 2021), the strain from $M$. unguiculatus in Inner Mongolia (Neimeng 2021), $Y$. pestis strains isolated from Inner Mongolia in 2019, together with $Y$. pestis genomes in CPIN were compared by the genome-wide SNPs (2). As shown in Figure 1A, the strain isolated from the patient and the strain isolated from M.unguiculatus 80 meters away from the houses of patient were clustered as $2 . M E D 3 q$ lineage, a lineage inherently belonging to the $Y$. pestis in the Erdos Plateaus M. unguiculatus plague focus in Inner Mongolia in 2019 (2).

In this $Y$. pestis infection event, the patient was a shepherd, and the family of the patient lived in a comparatively independent house that was located on the Ordos Plateaus $M$. unguiculatus plague focus in Inner Mongolia. There were a total of six families together with the patient's family within a threekilometer radius away from the house of the patient. According to reports from local residents, in the past two months, several dead $M$. unguiculatus had been found in the region before the case of human plague occurred. Because there were a total of four and three human plague cases occurred in the Inner Mongolia in 2019 and 2020, respectively, with 1 and 2 deaths in 2019 and 2020, respectively, and there were $2 Y$. pestis strains isolated from $M$. unguiculatus plague focus in Otog Qi in 2019, thus regulations were performing in Inner Mongolia that the local residents should be alerted to dead reservoirs and were required to report to local CDCs once the affected areas were found. The family of patient just had moved from Ningxia and been employed by a local inhabitant for only one year, so they did not know of the regulations and neglected to report dead rat phenomenon. Other five families' residents reported the abnormal dead rats phenomenon, and local professional staff conducted rodenticides in residential surrounding areas, but nothing less than the areas patient lived in was left.

After the human plague was confirmed, an enhanced rodent surveillance campaign was performed. A total of 2 recently dead $M$. unguiculatus were found, and the $Y$. pestis strain was isolated from each dead rat, with one just 80 meters away from the house of patient; in addition, over 70 fleas were found on the rat. In addition, there were a large number of fleas inhabiting those reported reservoirs. Such an observation indicated that serious $M$. unguiculatus plague epizootics with plenty of fleas existed in the patient's living areas and that the patient might have been infected from a flea bite.

\section{PUBLIC HEALTH RESPONSE}

First, before the 55-year-old patient was diagnosed as having plague, she sought treatment in 4 clinics or hospitals in Inner Mongolia and Ningxia. Therefore, it is critical to strengthen professional training for local clinicians in order to recognize and identify the disease earlier and be alerting to various forms of plague. In addition, the patient suffered from bubonic plague with secondary septicemic plague; unlike the pneumonic plague, the bubonic and the septicemic plague has limited the ability of person-to-person transmission (3). Therefore, more reasonable public health measures should be recommended in affected areas.

Secondly, since the human plague cases occurred in 2019 and 2020, the local CDCs had offered the local farmers or shepherds a Health Box [which included insect repellent (DEET), thermometer and report card et al.]. Corresponding educational efforts should be enhanced to promote behaviors such as wearing long pants and applying DEET to anyone engaged in outdoor activities in plague focus area; reporting rodent die-offs; avoiding direct contact with sick or dead wild animals (e.g., foxes or rabbits); using insecticides and rodenticides to eliminate the fleas and the host animals simultaneously, or insecticide be priority to animal plague control.

Thirdly, the Ordos Plateaus M. unguiculatus plague focus in Inner Mongolia is also adjacent to Ningxia 


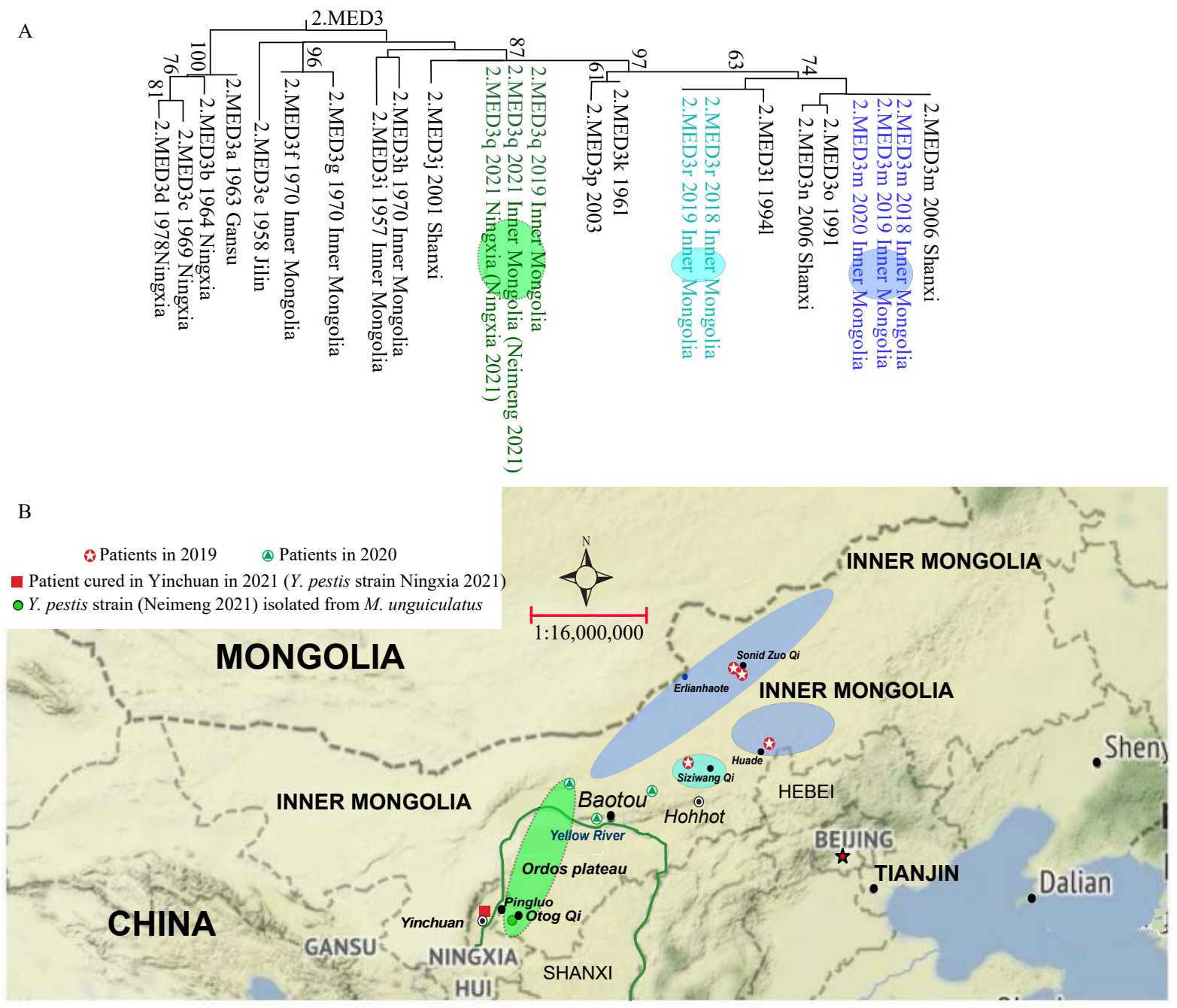

FIGURE 1. Geographical distribution and phylogenetic relationship of patients and various epizootics in Inner Mongolia and Ningxia. (A) Phylogenetic relationship based on whole genomic SNPs in Y. pestis 2. MED3 population. (B) Geographical distribution of phylogenetic lineages in Inner Mongolia.

Note: The maximum likelihood tree in Figure $1 \mathrm{~A}$ was constructed by the concatenated SNP using MEGA (Molecular Evolutionary Genetics Analysis Version 6.0. Tamura K, Stecher G, Peterson D, Filipski A, and Kumar S, 2013). Strains in colored circle on evolutionary trees isolated from the sites with same color on the map.

and Shanxi provincial-level administrative divisions (PLADs) in China (Figure 1B). There are no obvious geographic barriers between the $M$. unguiculatus plague focus in Inner Mongolia and its counterpart area in adjacent PLADs, so the joint prevention and control, including early joint warning and risk communication are necessary.

In addition, in the process of responding to the human plague events in China, the clinics commonly responsible for finding and treating patients, while the various level CDCs confirmed the diagnosis according to evidence based on laboratory assays. In this event, the biochemical analyzer in GHNMU reported that bacteria in the patient's blood were of the Yersinia genus, even though it did not definitively that the sample was $Y$. pestis (due to lacking a corresponding database of bacteria), the clinicians began to suspect that the patient might have plague. It was the second time that such situation had occurred in China as the first occurred in Yunnan Province in 2016 (1). In fact, with the gains in technical competence in hospitals, there are many techniques such as the genome sequencing, biochemical analyzers, and massspectrometric techniques, that can firstly give the clinicians the clues to plague.

\section{DISCUSSION}

The M. unguiculatus plague focus in Inner Mongolia 
can be divided into two parts: the Ordos Plateaus and the desert steppe of the Ulanqab plateaus. Previous studies provided insight into the relationship between plague intensity and the level of precipitation in the semi-arid grasslands of Inner Mongolia (4). i.e., Plague epizootics depend on changes in the density and distribution of local $M$. unguiculatus (3), while elevated rainfall facilitates increasing population levels of $M$. unguiculatus (4). A great increase in the local rodent population was an obvious feature in Inner Mongolia M. unguiculatus plague focus in 2019 (2). In 2019, a comparatively higher density $\left(3.1 / \mathrm{hm}^{2}\right)$ of the major host in this focus than the historical average level $\left(2 / \mathrm{hm}^{2}\right)$ on the Inner Mongolia Plateau (5) was observed. In 2021, drought was the main feature in Ordos Plateaus. The density of the main host reached to $4.46 / \mathrm{hm}^{2}$, and high flea density is a major feature of Otog Qi in August, such as the percentage of host infested flea was $52.63 \%$ with host-flea index was 3.93 in Otog Qi in August 2021. Similar situations could be observed in the adjacent Pingluo county, the percentage index and host-flea index were $59.26 \%$ and 2.15, respectively, in August (local plague surveillance data). Such ecological factors, i.e., higher flea density, also aroused a more potent animal plague epidemic with more risk of human infection.

\section{EPIZOOTICS OF PLAGUE IN 2018 TO 2021 IN INNER MONGOLIA}

Plague is primarily a disease of wild rodents. Animalto-animal transmission is mediated by flea bites, while human infection is often an accidental event, including being infected by the bites of escaped bacteriumbearing fleas. Continuous animal plague had occurred in previous years (in 2018, 2019, and 2020) in the $M$. unguiculatus Plague Focus in Inner Mongolia, and corresponding surveillance results indicated that animal plague epizootics were still active in the $M$. unguiculatus Plague Focus in Inner Mongolia (National Plague Surveillance report).

The $Y$. pestis strains inhabiting Inner Mongolia belong to 2.MED3 population. While $Y$. pestis strains in 2.MED3 population were further divided into various lineages (2.MED3a-p) in Inner Mongolia or adjacent PLADs such as Shanxi, Hebei, and Ningxia in China (6) (Figure 1). The $Y$. pestis strains isolated in 2018 and 2019 in Inner Mongolia could be divided into 3 lineages (2.MED3m, 2.MED3q, 2.MED3r) (2). Previous research found lineage $2 . \mathrm{MED} 3 \mathrm{~m}$ was a major lineage generally and affected most of the geographical area in Inner Mongolia $M$. unguiculatus plague focus in 2018 and 2019 (2). However, the patient-related stain was $2 . M E D 3 q$ lineage, which was mainly located in the Ordos Plateaus $M$. unguiculatus Plague Focus in Inner Mongolia (2).

Conflicts of interest: No conflicts of interest.

Acknowledgements: $\mathrm{Mu}$ Guo from Yunnan Institute for Endemic Disease Control and Prevention; Colleagues from China CDC, Ningxia CDC, and Inner Mongolia CDC.

Funding: The Key Bio-safety Research and Development Technology Project (2021YFC1200200), The Key Scientific and Technology Project of Inner Mongolia Autonomous Region (2021ZD0006).

\section{doi: $10.46234 / \mathrm{ccdcw} 2021.267$}

* Corresponding authors: Kuidong Shao, shaokuidong001@163.com; Tao Zhang, melody68man@163.com; Jianyun Li, hhhtlijianyun@ 163.com; Yumeng Wang, Wangyumeng@icdc.cn.

\footnotetext{
Center for Disease Control and Prevention of Ningxia Hui Autonomous Region, Yinchuan, Ningxia Hui Autonomous Region, China; ${ }^{2}$ General Center for Disease Control and Prevention of Inner Mongolia Autonomous Region, Huhhot, Inner Mongolia Autonomous Region, China; ${ }^{3}$ The Base for Control and Prevention of Plague and Brucellosis, Chinese Center for Disease Control and Prevention, Baicheng, Jilin, China; ${ }^{4}$ Beijing Ditan Hospital, Capital Medical University, Beijing, China; ${ }^{5}$ National Institute for Communicable Disease Control and Prevention, Chinese Center for Disease Control and Prevention, Beijing, China; ${ }^{6}$ State Key Laboratory of Infectious Disease Prevention and Control, Beijing, China.

${ }^{\&}$ Joint first authors.
}

Submitted: August 28, 2021; Accepted: December 21, 2021

\section{REFERENCES}

1. Shi LY, Yang GR, Zhang ZK, Xia LX, Liang Y, Tan HL, et al. Reemergence of human plague in Yunnan, China in 2016. PLoS One 2018;13(6):e0198067. http://dx.doi.org/10.1371/journal.pone.0198067.

2. Li JY, Wang YM, Liu F, Shen XN, Wang YT, Fan MG, et al. Genetic source tracking of human plague cases in Inner Mongolia-Beijing, 2019 PLoS Negl Trop Dis 2021;15(8):e0009558. http://dx.doi.org/10.1371/ journal.pntd.0009558.

3. Morelli G, Song YJ, Mazzoni CJ, Eppinger M, Roumagnac P, Wagner $\mathrm{DM}$, et al. Yersinia pestis genome sequencing identifies patterns of global phylogenetic diversity. Nat Genet 2010;42(12):1140 - 3. http://dx.doi. org/10.1038/ng.705.

4. Xu L, Liu QY, Stige LC, Ben Ari T, Fang XY, Chan KS, et al. Nonlinear effect of climate on plague during the third pandemic in China. Proc Natl Acad Sci USA 2011;108(25):10214 - 9. http://dx.doi.org/10.1073/ pnas. 1019486108.

5. Liu JY, Zhang WR. Inner Mongolia plague. Inner Mongolia autonomous region, China. Hohhot: Inner-Mongolia People Publishing House. 1997. (In Chinese).

6. Cui YJ, Yu C, Yan YF, Li DF, Li YJ, Jombart T, et al. Historical variations in mutation rate in an epidemic pathogen, Yersinia pestis. Proc Natl Acad Sci USA 2013;110(2):577 - 82. http://dx.doi.org/10.1073/ pnas. 1205750110. 